\title{
A short review on computational issues arising in relativistic atomic and molecular physics
}

\author{
Maria J. Esteban \\ Abstract. This paper is a short review of computational issues arising when \\ trying to compute electronic wave functions in atoms or molecules containing \\ heavy nuclei. In this case, relativistic effects can play an important role and \\ ad hoc models have to be used. The basic operator of this theories is the Dirac \\ operator, which unlike th Schrödinger operator, is unbounded both from below. \\ This feature creates dificulties both at the theoretical and the computational \\ levels. In this review we discuss some of these issues. A much detailed review \\ about this topic can be found in $[7]$.
}

\section{Introduction}

Quantum Mechanics and Quantum Chemistry are large field of investigation for the improvement of numerical and computational methods: the models involved are quite complex and so the computations are slow, heavy and often not accurate enough. Computing the electronic structure of an atom can be heavy, depending on the number of electrons involved, but the problems are still not too big. In the case of a large molecule, the number of electrons can be very large and moreover, there are nuclear singularities at different locations. But all these complications become much more delicate and heavy when one deals with relativistic computations. The relativistic effects are not negligible as soon as heavy nuclei are present in the atom or the molecule, and taking then into account often means replacing the Schrödinger operator with the Dirac operator. This operator is unbounded from below and electronic energies are to be found in the gap of its continuous spectrum. Minimization procedures leading to electronic energies in the nonrelativistic case should now be replaced by min-max procedures and this is quite difficult to implement at the numerical level. So, other alternatives have to be found to deal with the unboundedness of the operator.

The basic theory in relativistic quantum mechanics is QED (Quantum Electrodynamics) but this is too complicated for computations, except in very simple situations. So, for an $N$-electron system the most commonly used models used in numerical computations are based on the Hamiltonian

1991 Mathematics Subject Classification. Primary 47A75, 81V45, 81V55; Secondary 65N25, 35Q40, 35Q75, 47A11, 47N50, 49R50.

Key words and phrases. Dirac operator, Dirac-Fock equations, eigenvalues, gap of the essential spectrum, bound states, relativistic equations.

MJE aknowledges partial support of European Programs HPRN-CT \# 2002-00277 \& 00282. 


$$
H=-\sum_{i=1}^{N}\left(D_{c, x_{i}}+V\left(x_{i}\right)\right)+\frac{1}{2} \sum_{\substack{i, j=1 \\ i \neq j}}^{N} \frac{1}{\left|x_{i}-x_{j}\right|},
$$

where $D_{c, x_{i}}$ is the Dirac operator acting on the $x_{i}$ variable:

$$
D_{c}=-i c \hbar \alpha \cdot \nabla+m c^{2} \beta=-i c \hbar \sum_{k=1}^{3} \alpha_{k} \partial_{k}+m c^{2} \beta .
$$

The usual representation of the matrices $\alpha_{1}, \alpha_{2}, \alpha_{3}$ and $\beta$ in $2 \times 2$ blocks is given by

$$
\beta=\left(\begin{array}{cc}
I_{2} & 0 \\
0 & -I_{2}
\end{array}\right), \quad \alpha_{k}=\left(\begin{array}{cc}
0 & \sigma_{k} \\
\sigma_{k} & 0
\end{array}\right) \quad(k=1,2,3),
$$

where the Pauli matrices $\sigma_{k}$ 's are defined as

$$
\sigma_{1}=\left(\begin{array}{ll}
0 & 1 \\
1 & 0
\end{array}\right), \quad \sigma_{2}=\left(\begin{array}{cc}
0 & -i \\
i & 0
\end{array}\right), \quad \sigma_{3}=\left(\begin{array}{cc}
1 & 0 \\
0 & -1
\end{array}\right) .
$$

On the other hand, $V$ is the nuclear potential:

$$
V(x)=\sum_{j=1}^{M} \frac{Z_{j}}{\left|x-X_{j}\right|} .
$$

$M$ being the number of nuclei and $Z_{j}$, the charge of the $j$-th nuclei, which is supposed to be fixed at the point $X_{j}$. Note that this model supposes that the nucleii are point-like. One can also consider extended nucleus models in which the support of the nuclear distribution of charge contains an open set. Actually, modern atomic and molecular relativistic calculations often use finite nuclear models, see for instance reference [51].

As far as this review is concerned, the main properties satisfied by the operator $D_{c}$ are:

$$
D_{c}^{2}=-c^{2} \hbar^{2} \Delta+m^{2} c^{4},
$$

and the fact that the spectrum of $D_{c}$ is unbounded both from above and below and has a gap between $-m c^{2}$ and $m c^{2}$ :

$$
\sigma\left(D_{c}\right)=\left(-\infty,-m c^{2}\right] \cup\left[m c^{2},+\infty\right) .
$$

Note that the operator $H$ acts of functions $\psi: \mathbb{R}^{3} \rightarrow \mathbb{C}^{4}$, the so-called 4-spinors. Let us write 4-spinors in the form $\psi=\left(\begin{array}{l}\varphi \\ \chi\end{array}\right), \varphi, \chi: \mathbb{R}^{3} \rightarrow \mathbb{C}^{2}$. In what follows we will often refer to the $\varphi$ (resp. $\chi$ ) as the upper or larger (resp. lower or smaller) spinor.

In the choice of the above Hamiltonian there are a number of simplifications which have been made: the nuclei are supposed to be point-like and all electrostatic interactions (electron-electron and nuclei-electrons) are described as Coulomb interactions. Moreover, this Hamiltonian is not Lorentz invariant as any correct relativistic theory should be. Nevertheless this model seems to describe quite accurately the electrodynamics of an atom or a molecule. Note also that appart from the above questions, there are other issues related to the use of this Hamiltonian. The first one is that the whole real line is in its spectrum, and so there cannot be stable bound states, since all possible eigenvalues would be embedded in the continuum. The second issue is related to the so-called variational collapse. This means that computations can be easily lead to very negative values for the energies, 
since $D_{c}$ is unbounded from below. Finally, the appearance of spurious eigenvalues in the implementation of many available numerical methods is also of concern.

The operator $H$ acts on functions which are defined in $\mathbb{R}^{3 N}$ and with values in $\mathbb{C}^{4}$. So, as soon as $N$ is not too small, computations are almost impossible to be performed. One of the solutions found in Physics and Chemistry to deal with this difficulty is to consider functions in a smaller space. More concretely, since the Pauli Principle forces electronic wavefunctions to be antisymmetric, Swirles [48] proposed to look for wave functions which are stationary with respect to the Hamiltonian $H$ in the space of the so-called Slater determinants (note that the first to have proposed such a procedure in the non relativistic context was Hartree). With this procedure, called the Dirac-Fock approximation, one computes an approximation of the eigenvalues of $H$ by restricting the quadratic form $\Psi \mapsto\langle\Psi, H \Psi\rangle$ to the class of the functions $\Psi$ which are a simple Slater determinant:

$$
\Psi=\psi_{1} \wedge \cdots \wedge \psi_{N}
$$

where $\left(\psi_{1}, \ldots, \psi_{N}\right)$ is an orthonormal system of $L^{2}\left(\mathbb{R}^{3}, \mathbb{C}^{4}\right), \int_{\mathbb{R}^{3}}\left(\psi_{i}, \psi_{j}\right)_{\mathbb{C}^{2}}=\delta_{i j}$. The precise meaning of (1.6) is:

$$
\Psi_{k}\left(x_{1}, \ldots, x_{N}\right)=\frac{1}{\sqrt{N !}} \operatorname{det}\left(\psi_{i, k}\left(x_{j}\right)\right), \quad k=1, \ldots, 4, \quad i, j=1, \ldots, N .
$$

Since the set of all the $\Psi$ 's having the form (1.6) is not a vector subspace of $\bigwedge_{i=1}^{N} L^{2}\left(\mathbb{R}^{3} \times\{ \pm\}, \mathbb{C}^{4}\right)$, one then obtains an energy functional which is nonlinear in terms of $\psi_{1}, \ldots, \psi_{N}$. The associated Euler-Lagrange equations form a system of $N$ coupled nonlinear PDEs, the so-called DF (Dirac-Fock) equations, which can be written as follows

$$
D_{c, \psi} \psi_{k}=\epsilon_{k} \psi_{k}, \quad k=1, \ldots, N,
$$

where $\psi=\left(\psi_{1}, \ldots, \psi_{N}\right)$ satisfies $\int_{\mathbb{R}^{3}}\left(\psi_{i}(x), \psi_{j}(x)\right) d x=\delta_{i j}$, i.e.

$$
\operatorname{Gram}(\psi)=\mathbb{1},
$$

and

$$
\gamma_{\psi}(x, y)=\sum_{\ell=1}^{N} \psi_{\ell}(x) \otimes \psi_{\ell}(y)^{*}, \quad \rho_{\psi}(x)=\operatorname{tr}_{\mathbb{C}^{4}}\left(\gamma_{\psi}(x, x)\right)=\sum_{\ell=1}^{N}\left(\psi_{\ell}(x), \psi_{\ell}(x)\right) .
$$

Equations (1.7) are the Euler-Lagrange equations of the Dirac-Fock functional

$$
\begin{aligned}
\mathcal{E}_{\mathrm{DF}}^{\nu, c}(\psi)=\sum_{\ell=1}^{N}\left(\psi_{\ell}, D_{c} \psi_{\ell}\right)_{L^{2}} & -\sum_{\ell=1}^{N}\left(\psi_{\ell},\left(\nu * \frac{1}{|x|}\right) \psi_{\ell}\right)_{L^{2}} \\
+ & \frac{1}{2} \iint_{\mathbb{R}^{3} \times \mathbb{R}^{3}} \frac{\rho_{\psi}(x) \rho_{\psi}(y)-\operatorname{tr}\left(\gamma_{\psi}(x, y) \gamma_{\psi}(y, x)\right)}{|x-y|} d x d y
\end{aligned}
$$

on the manifold

$$
\mathcal{M}_{\mathcal{D F}}:=\left\{\psi=\left(\psi_{1}, \ldots, \psi_{N}\right) \in\left(H^{1 / 2}\left(\mathbb{R}^{3}, \mathbb{C}^{4}\right)^{N}, \quad \operatorname{Gram}(\psi)=\mathbb{1}\right\} .\right.
$$


Using rather complicated variational methods, the existence of solutions to (1.7) when $Z:=\sum_{j=1}^{M} Z_{j}>N-1$ has been proved in $[\mathbf{1 8}, 44]$. But in this topic the theoretical issues of existence are not, for the moment, very linked to the computational issues, except in the case $N=1$, where interesting computational methods have been found recently by using the variational structure highlighted by the theoretical existence results (see Section 3).

The Dirac-Fock equations seem to give an accurate description of the electronic structure in closed-shell ${ }^{1}$ atoms, but neither in open-shell atoms, nor in molecules. For this, the MCDF (multi-configuration Dirac-Fock) equations are more appropriate: here the idea is not to work in the set $\mathcal{M}_{D F}$ of the Slater determinants, but rather consider well-chosen linear combinations of Slater determinants. The idea here is the following : in order to construct a basis of Slater determinants, one chooses as basis functions for the $\psi_{i}$ 's the eigenfunctions of the operator $D_{c}+V$. These eigenfunctions correspond to different eigenvalues and have attached to them some numbers, denoted by $\Pi, J, M$, corresponding to parity and angular momentum. Multiconfiguration methods consider linear combinations of all Slater determinants which have the same numbers $\Pi, J$ and $M$.

In the coming sections we present some of the methods used to compute approximate solutions for the above models. Section 2 deals with the general case $M \geq 1, N \geq 1$, while Section 3 is devoted to the monoelectronic case : $M \geq 1$, but $N=1$.

\section{Computations for atoms and molecules : the multi-electronic case.}

Form the computational viewpoint, the difference between the DF and the MCDF equations is that the latter are more complicated and cannot be diagonalized as the former ones. But regarding the numerical work needed to solve them, they are quite similar in structure and properties. So, in this section we will deal with both of them at the same time.

Up to now there are two basic methods to solve numerically the MCDF equations : either by using analytical or numerical basic sets and then perform a Galerkin approximation, or find directly a numerical solution of the equations by discretizing them. It seems that the direct numerical resolution is better suited for small systems, but for cases with millions of determinants ( $N$ very large) analytical basis set techniques seem better suited.

The methods based on discretization of the equations are mostly predictorcorrector or finite difference methods, but recently there have been also some works based on finite element methods. The main idea is to solve the highly nonlinear equations by an iteration procedure (a fixed-point argument): at every step of the iteration, the eigenvalues correspond to the Hamiltonian constructed with the eigenfunctions obtained at the previous step. Much of the work found in the Physics and Chemistry litterature is concerned with the calculation of the coefficients of the different terms, that is with the algebraic structure of the system of unknowns. This reduces the number of terms to be computed numerically to the strict minimum, which has a consequence the reduction of the numerical errors. Good references for this are $[\mathbf{6}, \mathbf{2 1}, \mathbf{2 3}]$.

\footnotetext{
${ }^{1}$ Closed shells are the shell filled with the maximum number of electrons as allowed by the Pauli principle (see [50])
} 
In order to deal with the singularities created by those of the nuclear potential and to get better accuracy for the eigenfunctions in the region where they are singular, the equations are often discretized after a logarithmic change of variables has been performed around the singularities. In the diatomic case, the elliptic hyperbolic coordinates are often used to reduce the complexity and better discretize the problem around the two singularities. In more complex molecules little can be done to reduce the numerical work.

Good general references about the numerical methods presented above, their specificities, and some comparison between them can be found in Chapters 3 and 4 of [7]. In particular, one can see the reasons why predictor-corrector methods can have some interest to treat part of the system, but cannot avoid some problems that finite difference schemes can. Some algorithms are mixed predictor-corrector/finite differences.

Some important difficulties appearing in many of the existing algorithms are: keeping the continuity of some of the solution's components, speeding the sometimes very slow convergence rate, avoiding (or dealing with) spurious roots and most of all, not falling into the negative continuum (the so-called variational collapse that "haunts" all people making relativistic computations). One of the solutions proposed by some authors $[\mathbf{3 1}]$ is to consider ad hoc projector operators to avoid too negative energies. Let us discuss this issue a little bit in detail, since it is of major importance in relativistic computations (and absent in nonrelativistic ones). Avoiding negative energies is often interpreted as avoiding positronic states. When trying to obtain a Hamiltonian from QED one sees that electronic states appear as eigenfunctions of a Hamiltonian which contains the Dirac operator but "sandwiched" between two projector operators, which project onto the positive energy space. These operators are self-consistent (depend on the solutions) and so they are not known a priori. Taking the projectors associated to the free Dirac operator ( i.e. considering the so-called Brown-Ravenhall operator) does not seem a good idea, since the real projectors depend strongly on the external potential. Constructing the projection operator using the solutions of the free-particle Dirac equation introduces errors, as pointed out in [29]. Many propositions have been made and we will discuss them more in detail in the next section. Mittleman [43] suggested using continuously updated projection operators in SCF-calculations. In the finitebasis (algebraic) approximation such projection operators are implicitly used by selecting for the next iteration the lowest positive-energy solutions. Correct convergence is then straightforwardly obtained by combining such vector selection with kinetic balance. In [31] it has been proposed to use positive projection operators corresponding to finite basis composed of the eigenfunctions of a simpler problem, but many options are possible, like for instance choosing well the boundary conditions in the discretized problem, which is in fact a similar projection procedure and works well. On the other hand, when using basis sets techniques, it is clearly much easier to "project out" the very negative energies, since the choice of the basis (the behaviour of the basis elements at infinity for instance) determines the space of the final solutions, and therefore, their behavior also. A final (very extended and used) technique to avoid the variational collapse, is to use the so-called kinetic-balance principle (see $[\mathbf{3 7}, \mathbf{4 7}]$ ), which forces the spinor lower component to have a specific form (as a function of the upper spinor). This is somehow equivalent to using a Brown-Ravenhall operator, since the link between the upper and the lower spinors 
are the ones satisfied by (generalized) eigenfunctions of the free Dirac operator. When solving the Dirac equation or mean-field generalisations of it for atoms by finite-difference methods, spurious solutions are avoided by imposing the correct boundary conditions near the origin and near infinity in the radial equations. In molecular calculations using basis set expansions the correct coupling of the large and small components are obtained by employing the kinetic balance relation (see references above). In the next section we explain in detail a recent method allowing to avoid the variational collapse when $N=1$ hoping that this sheds some light on the general problem.

Let us add that negative eigenvalues do not necessarily correspond to positronic states, and that one can have negative electronic states in molecules. So, all the efforts to avoid these negative eigenvalues have to be weighed carefully, determining in which context the measures taken are needed and/or useful.

Regarding molecular computations, most of the existing methods are based on the use of basis sets (a Galerkin-like method), the basis sets being analytical or numerical. The former allow to perform many computations exactly or independently before launching the final numerical computation (for a good description see [46] and more in particular the article of K. Faegri and K.G. Dyall in [46]). Their use and choice relies on a sound chemical knowledge about the solutions' properties. Note that it is quite usual for molecular calculations to avoid the variational collapse by defining the small component in terms of the large one [37], by using for instance the kinetic-balance principle, which is not very accurate but helps to avoid variational collapse.

A good discussion about the appearance of spurious solutions in the molecular computations and also about the possible reasons for poor convergence (or lack of convergence in some cases) is found in $[45, \mathbf{1 6}]$.

The question of how to choose a good basis set has been addressed in many papers, for instance in $[\mathbf{1 2}, \mathbf{1 3}, \mathbf{2 0}, \mathbf{2 2}, \mathbf{3 2}, \mathbf{3 7}]$.

Finally note that there exist some packages to perform full relativistic atomic and molecular computations, as GRASP (http://web.am.qub.ac.uk/DARC/grasp.html), BERTHA (see [24]), DIRAC (http://dirac.chem.sdu.dk/) and of course, the first one to have existed, due to Desclaux [6]. A more recent, but quite complete code is the UTCHEM code (http://utchem.qcl.t.u-tokyo.ac.jp/).

\section{Computations in the case of a single electron.}

The case of a single electron seems quite simple at first sight, since the problem is now to find eigenvalues and eigenfunctions of operators $D_{c}+V$ where $V$ is an external electrostatic potential, which is in general nonpositive: in atomic and molecular physics, $V$ stands in general for a nuclear potential, and it is in general nonpositive, i.e. attractive. We are interested in eigenvalues not lying in the continuum spectrum, since they are supposed to represent stable bound state energies. If the Dirac operator were bounded from below this question would be quite simple to deal with, both analytically and numerically, since the ground state energy, $\lambda_{1}(V)$, if it existed, would be the minimum of the Rayleigh quotient

$$
R_{V}(\psi):=\frac{\left(\psi,\left(D_{c}+V\right) \psi\right)}{(\psi, \psi)} .
$$

The other eigenvalues above $\lambda_{1}(V)$ would be obtained as minimizers of the Rayleigh quotient on the orthogonal space of the previously found eigenfunctions. 
But in the case of the Dirac operator, its unboundedness from below forces us to look for eigenvalues in gaps of the continuum (essential) spectrum, and therefore, no eigenvalues can be found by a global minimization of $R_{V}$ since the infimum of $R_{V}$ is equal to $-\infty$.

When the potential $V$ is not too singular (and we are going to assume this always from now on), the essential spectrum of the operator $D_{c}+V$ is, as for the free operator $D_{c}$, the set $\left(-\infty,-m c^{2}\right] \cup\left[m c^{2},+\infty\right)$. So, in a time independent model eigenvalues corresponding to stable bound states of an electron in the electrostatic field corresponding to the potential $V$ have to be in the gap $\left(-m c^{2}, m c^{2}\right)$ and formally correspond to min-max values of the Rayleigh quotient $R_{V}$ (note that in a time dependent model, eigenvalues can enter the gap by its both ends). This problem is not easy to solve, and different ways have been explored so far. Indeed, even if this case seems not very interesting because few atoms and molecules have a single electron, the linear Dirac equation appears also when dealing with the multielectronic case and solving the corresponding equations by a iterative procedure (see Section 2). Hence, this problem has attracted a lot of attention both in the Physics and in the Chemistry litterature, as a first (and often intermediate) step to deal with real and complex atoms and molecules.

As in the multielectron case, various alternative approaches have been used: variational methods, basis set techniques or derivation of effective bounded-frombelow operators which in some sense approach well the unbounded Dirac operator. From the mathematical viewpoint little had been done until 10 years ago when rigourous variational (min-max) approaches were proposed to characterize the eigenvalues $[\mathbf{1 7}, \mathbf{2 6}, \mathbf{2 5}, \mathbf{8}, \mathbf{9}]$. Soon after, efficient and robust algorithms based on some of the rigourous variational approaches were proposed $[\mathbf{1 1}, \mathbf{1 0}]$. In the remainder of this section we describe some of the existing approaches to compute the eigenvalues of operators of the form $D_{c}+V$.

At this point let us stress that in order to apply almost all that is going to be said below, we need to ask that the potential $V$ is not too singular, which means that it can have only a finite number of point singularities, and that around any of those singular points, $\bar{x}$, it has to behave less singularly than the Coulomb potential $-c /|x-\bar{x}|$. More precisely, we need that for all $\bar{x} \in \mathbb{R}^{3}$,

$$
\limsup _{x \rightarrow \bar{x}}|x-\bar{x}| V(x)<c .
$$

This condition is there to ensure that the operator $D_{c}+V$ has a well-defined selfadjoint extension. Moreover, without (3.2), the operator $D_{c}$ would not be able to control the potential $V$, what is necessary to define the min-max characterizations of the eigenvalues of $D_{c}+V$ (it is interesting to see the relation between this and a Hardy-like inequality that was proved in $[\mathbf{9}]$ ).

Coming back to the methods proposed to compute the eigenvalues, let us start their description by the ones consisting in a discretization of the problem in a finite basis set of dimension $n$. Once the basis is chosen, the problem consists, of course, in finding the eigenvalues of the correspoonding $n \times n$ matrix. The choice of the basis is crucial, since at a discrete level, if the basis set is just any, we will find eigenvalues corresponding to the discretization of the continuum, that is, above $m c^{2}$ and below $-m c^{2}$ and also eigenvalues in the gap $\left(-m c^{2}, m c^{2}\right)$. Only the latter are of interest to us. The efficiency of the method depends on the good choice of the basis set, and this efficiency is related to the behavior at infinity of the basis set 
functions and also to the sparseness properties of the discrete matrix. A difficulty that has been often encountered is the appearance of spurious eigenvalues, that is eigenvalues of the discretized $n \times n$ matrix which do not approach eigenvalues of the operator $D_{c}+V$ (see for instance $[\mathbf{1 2}]$ ). W. Kutzelnigg has written two excellent reviews $[\mathbf{3 7}, \mathbf{3 8}]$ about the use of basis sets in Dirac calculations, where many relevant references can be found. About this approach let us say that in the choice of the basis sets different strategies have been taken: either look for efficiency of the numerical method by choosing for instance B-splines [32], or choose analytical basis sets which help to compute some of the integrals almost explicitly and which represent well the expected behavior of the eigenfunctions at infinity $[\mathbf{1 2}, \mathbf{1 3}]$. About how to choose a good basis sets, see $[\mathbf{2 0}, \mathbf{2 2}, \mathbf{3 7}]$.

The second approach consists in searching for operators which approach the Dirac operator $D_{c}+V$ in some sense and which are bounded below. The real reason why this can work is that at the nonrelativistic limit (for $c$ going to $+\infty$ ), the spectrum of the operator $D_{c}+V$, shifted by $-m c^{2}$, is known to converge towards the spectrum of the Schrödinger operator $-\hbar^{2} \Delta / 2+V$, operator which is bounded from below if $V$ satisfies the conditions described above. A long series of papers propose to approach $D_{c}+V$, or operators which are spectrally equivalent to it, by operators which equal to $-\hbar^{2} \Delta / 2+V$ plus terms which are "small" when $c$ is large. The first series of works considers unitary transformations $W$ which do not change the spectrum, and writes down an expansion of $W^{\dagger}\left(D_{c}+V\right) W$ in powers of $c^{-2}$ :

$$
W^{\dagger}\left(D_{c}+V\right) W=H_{0}+\frac{H_{1}}{c^{2}}+\frac{H_{2}}{c^{4}}+\cdots+\frac{H_{k}}{c^{2 k}}+\cdots
$$

and cut it at a finite power $k \geq 1$. There are many ways to choose $W$, the different strategies trying to optimize some given criterium. In many of the proposed methods, $H_{0}=-\hbar^{2} \Delta / 2+V+m c^{2}$, and the operators $H_{1}, H_{2}, \ldots$ are easy to compute formally. Some of the approaches are aimed at diagonalizing the operator $D_{c}+V$. The first attempt in this direction was the so-called Foldy-Wouthuysen transformation [19] which is not known in closed form, but which can be again expanded in powers of $c^{-2}$. A very good discussion of approaches made in this direction can be found in [1]. Many other proposals have followed in order to avoid some of the problems encoutered by the Foldy-Wouthuysen transformation. Some of the above expansions present convergence problems near the singularities of the potential, and to remedy this, Chang et al [3], proposed a new method to write the expansion, called ZORA, convergent this time: the expansion is now made in powers of $\left(2 m c^{2}-V\right)^{-1}$ (see also $[\mathbf{1 4}, \mathbf{1 5}]$ ). A similar idea appears in the work of Heully et al $[\mathbf{2 8}, \mathbf{2 9}]$ and this method has been studied in detail by van Lenthe et al [41]. A good review about this kind of methods has been written by Kutzelnigg [39] and more recent proposals for exact 2-component relativistic Hamiltonians can be found in $[40]$.

Before we come to the third kind of strategy to find and compute the eigenvalues of Dirac operators, the variational one, let us remark that something that has been noticed by many people is that the eigenvalue problem

$$
\left(D_{c}+V\right) \psi=\lambda \psi
$$


is equivalent to an eigenvalue problem for the upper spinor $\varphi$, where $\psi=\left(\begin{array}{l}\varphi \\ \chi\end{array}\right)$, $\varphi, \chi: \mathbb{R}^{3} \rightarrow \mathbb{C}^{4}$. Indeed (3.4) is equivalent to the system

$$
\left\{\begin{array}{l}
R \chi=\left(\lambda-m c^{2}-V\right) \varphi \\
R \varphi=\left(\lambda+m c^{2}-V\right) \chi
\end{array}\right.
$$

with $R=-i c \hbar(\vec{\sigma} \cdot \vec{\nabla})=-i c \hbar \sum_{k=1}^{3} \sigma_{k} \partial / \partial x_{k}$ where $\sigma_{1}, \sigma_{2}, \sigma_{3}$ are the Pauli matrices defined in Section 1. Then, if $\psi$ is an eigenfunction of $H$ associated with the eigenvalue $\lambda$, and if the function $\lambda+m c^{2}-V$ is never equal to 0 , we have:

$$
\chi=\frac{R \varphi}{\lambda+m c^{2}-V},
$$

and

$$
R\left(\frac{R \varphi}{\lambda+m c^{2}-V}\right)+\left(m c^{2}+V\right) \varphi=\lambda \varphi
$$

The above equation can be seen as an eigenvalue problem for the eigenvaluedependent operator $H(\lambda):=R\left(\lambda+m c^{2}-V\right)^{-1} R+m c^{2}+V$ which acts on 2component functions, unlike the Dirac operator $D_{c}+V$ which acts on 4-component functions. And solutions of (3.7) are eigenfunctions corresponding to the zero eigenvalue for the operator $H(\lambda)-\lambda$. To those knowing about pencil problems, this is one of them.

For many years the eigenvalue dependence of $H$ has been an obstacle in the way of finding eigenvalues of Dirac operators. As we will see below, one can keep it and still derive from it a very good method to find and compute the Dirac eigenvalues. But this will be the case only for 1-electron systems!

The third way to find and compute the eigenvalues of operator $D_{c}+V$ is based on variational ideas. As we said above, minimimizing the Rayleigh quotient is out of question, since the operator is not bounded from below. A possibility is to minimize it in a set of functions in which it is bounded from below, that is, find a subspace which is large enough to contain, or almost contain, all the eigenfunctions, but not too large to allow the Rayleigh quotient to take values below $-m c^{2}$. This is tricky, specially because the space certainly depends on $V$. Other ways have been explored : to look for eigenvalues of $\left(D_{c}+V\right)^{2}[\mathbf{5 2}, \mathbf{2}]$ or of $\left(D_{c}+V\right)^{-1}[\mathbf{3 0}]$. The first idea is good if the potential $V$ does not support any negative eigenvalue, which is not always the case. Moreover, practically speaking, the integrals entering into the computation become much more difficult to compute. The second one needs that there are no eigenvalues of $D_{c}+V$ around 0 . And both would had difficulties to distinguish electronic from positronic states.

A last idea is to use the fact that the eigenvalues are actually stationary values (min-maxes) for the Rayleigh quotient, and under the appropriate assumptions on $V$, they correspond rigourously to min-max critical values for $R_{V}[\mathbf{1 7}, \mathbf{2 6}, \mathbf{2 5}, \mathbf{8}, \mathbf{9}]$. Computationally speaking, Talman [49] and Datta et al [5] seem to have been the first ones to have the idea of computing the ground-state energy for $D_{c}+V$ by taking a min-max of $R_{V}$ by first maximizing in the lower componer of the spinors $\psi$ and then minimizing in the upper component. The work in $[\mathbf{2 5}, \mathbf{9}]$ has showed that Talman's and Datta et al's idea can be made rigorous and that it actually leads to very efficient algorithms to compute the eigenvalues of $D_{c}+V$. Indeed, as shown in [9], for a giver upper spinor $\varphi: \mathbb{R}^{3} \rightarrow \mathbb{C}^{4}$, the maximum of $R_{V}$ over all 
possible lower spinors $\chi, \lambda(\varphi)$, satisfies a scalar equation :

$$
\lambda(\varphi) \int_{\mathbb{R}^{3}}|\varphi|^{2} d x=\int_{\mathbb{R}^{3}}\left(\frac{m c^{2} \hbar^{2}|(\sigma \cdot \nabla) \varphi|^{2}}{m c^{2}-V+\lambda(\varphi)}+\left(m c^{2}+V\right)|\varphi|^{2}\right) d x .
$$

The ground state energy is now the infimum of a function, $\lambda(\varphi)$ over all $\varphi$ 's, it is so the solution of a minimization problem which is well posed, since the function $\lambda(\varphi)$ is bounded from below. Discretizing this minimization procedure is now easy and it has been made in $[\mathbf{1 1}, \mathbf{1 0}]$. Later on Kolb et al have used finite element and LCAO (linear combination of atomic orbitals) methods to solve the same problem $[34,35,36,55]$. In our opinion Talman's paper [49] has played a key role in realizing the min-max nature of the Dirac eigenvalue problem.

This method works perfectly, without fear of variational collapse or other diseases present in most computations in relativistic quantum mechanics. But only for potentials which are attractive (this is not a drawback in applications, since nuclear potentials are attractive). The main drawback is that it is not clear how to export the underlying ideas to the multielectronic case, except if one considers DFT (density functional theory) or OEP (optimized effective potential) models, in which all the potentials are local, even those modelling inter-electronic interaction.

Note that this method is directly related to the scalar nonlinear problem (3.7), that is, to the search of $\lambda$ 's such that 0 is an eigenvalue of $H(\lambda)-\lambda$. This is the eigenvalue-dependent operator that so many people have tried to avoid. In this method, we do not avoid it, on the contrary we fully use it to obtain an exact minimization characterization for the ground state and good and efficient minimization algorithms for its computation. Actually, we discovered recently that this method is equivalent to a method used by van Lenthe et al [42] (see also [27]) to solve (3.7) in an iterative way. In this paper they give computational results showing that the method is quite effective, even if not much better than ZORA except for deep core electrons. Some final references for eigenvalue-dependent 2component Hamiltonian are due to Cowan and Griffin [4] in the framework of the Hartree-Fock model and to Wood and Boring [54] in a DFT framework. A recent version was proposed by Malkin and co-workers [33].

Aknowledgement. The author would like to thank the referee for very useful and interesting comments and new references.

\section{References}

[1] M. Barysz, A. Sadlej. Two-component methods of relativistic quantum chemistry: from the Douglas-Kroll approximation to the exact two-component formalism. J. Mol. Struct. (Theochem) $\mathbf{5 7 3}$ (2001), p. 181-200.

[2] W.E. Bayliss, S. J. Peel. Stable variational calculations with the Dirac Hamiltonian. Phys. Rev. A, 28 (4) (1983), p. 2552-2554.

[3] Ch. Chang, J.-P. Pelissier, Ph. Durand. Regular two-Component Pauli-like Effective Hamiltonians in Dirac Theory. Phys. Scripta 34 (1986), p. 394-404.

[4] R. D. Cowan, D.C.Griffin. Approximate relativistic corrections to atomic radial wave functions. J. Opt. Soc. Am. 66 (1976), p. 1010.

[5] S.N. Datta, G. Deviah. The minimax technique in relativistic Hartree-Fock calculations. Pramana 30(5) (1988), p. 393-416.

[6] J. P. Desclaux. A Relativistic Multiconfiguration Dirac-Fock Package. In Methods and Techniques in Computational Chemistry Clementi. E. Ed A: Small Systems STEF, 1993. 
[7] J.P. Desclaux, J. Dolbeault, M.J. Esteban, P. Indelicato, E. Séré. Computational approaches of relativistic models in quantum chemistry. In Handbook Numer. Anal., X, p. 453-483, NorthHolland, Amsterdam, 2003.

[8] J. Dolbeault, M.J. Esteban, E. Séré. Variational characterization for eigenvalues of Dirac operators. Calc. Var. and P.D.E. 10 (2000), p. 321-347.

[9] J. Dolbeault, M.J. Esteban, E. Séré. On the eigenvalues of operators with gaps. Application to Dirac operators. J. Funct. Anal. 174 (2000), p. 208-226.

[10] J. Dolbeault, M.J. Esteban, E. Séré. A variational method for relativistic computations in atomic and molecular physics. Int. J. Quantum. Chemistry 93 (2003), p. 149-155.

[11] J. Dolbeault, M.J. Esteban, E. Séré, M. Vanbreugel. Minimization methods for the oneparticle Dirac equation. Phys. Rev. Letters 85 (19) (2000), p. 4020-4023

[12] G.W.F. Drake, S.P. Goldman. Application of discrete-basis-set methods to the Dirac equation. Phys. Rev. A 23 (1981), p. 2093-2098.

[13] G.W.F. Drake, S.P. Goldman. Relativistic Sturmian and finite basis set methods in atomic physics. Adv. Atomic Molecular Phys. 23 (1988), p. 23-29.

[14] Ph. Durand. Transformation du Hamiltonien de Dirac en Hamiltoniens variationnels de type Pauli. Application à des atomes hydrogenoïdes. C. R. Acad. Sc. Paris $\mathbf{3 0 3}$, série II, numéro 2 (1986), p. 119-124.

[15] Ph. Durand, J.-P. Malrieu. Effective Hamiltonians and pseudo-potentials as tools for rigorous modelling. In Ab initio methods in Quantum Chemistry I. K.P. Lawley ed.. J. Wiley \& Sons, 1987.

[16] K.G. Dyall, I.P. Grant, S. Wilson. Matrix representation of operator products. J. Phys. B: At. Mol. Phys. 17 (1984), p. 493-503.

[17] M.J. Esteban, E. Séré. Existence and multiplicity of solutions for linear and nonlinear Dirac problems. Partial Differential Equations and Their Applications. CRM Proceedings and Lecture Notes, vol. 12. Eds. P.C. Greiner, V. Ivrii, L.A. Seco and C. Sulem. AMS, 1997.

[18] M.J. Esteban, E. Séré. Solutions for the Dirac-Fock equations for atoms and molecules. Comm. Math. Phys. 203 (1999), p. 499-530.

[19] L.L. Foldy, S.A. Wouthuysen. On the Dirac Theory of Spin 1/2 Particles and Its NonRelativistic Limit. Phys.Rev. 78 (1950), p. 29-36.

[20] I.P. Grant. Conditions for convergence of variational solutions of Dirac's equation in a finite basis. Phys. Rev. A 25(2) (1982), p. 1230-1232.

[21] I. P. Grant. Relativistic Atomic Structure Calculations. Meth. Comp. Chem. 2 (1987), p. 132.

[22] I.P. Grant. Notes on Basis Sets for Relativistic Atomic Structure and QED. A.I.P. Conf. Proc. 189 (1989), p. 235-253. Ed. P.J. Mohr, W.R. Johnson, J. Sucher.

[23] I. P. Grant, H. M. Quiney. Foundation of the Relativistic Theory of Atomic and Molecular Structure. Adv. At. Mol. Phys. 23(1988), p. 37-86.

[24] I. P. Grant, H. M. Quiney, H. Skaane. BERTHA - 4-component relativistic molecular quantum mechanics, in Supercomputers, Collision Processes and Applications, edited by K. L. Bell, K. A. Berrington, D. S. F. Crothers and K. T. Taylor, pp. 213-224 (Plenum, New York, 1999).

[25] M. Griesemer, R.T. Lewis, H. Siedentop. A minimax principle in spectral gaps: Dirac operators with Coulomb potentials. Doc. Math. 4 (1999), 275-283 (electronic).

[26] M. Griesemer, H. Siedentop. A minimax principle for the eigenvalues in spectral gaps. J. London Math. Soc. (2) 60 no. 2 (1999), p. 490-500.

[27] D. Hegarty. On the Dirac equation in the algebraic approximation. Theor. Chim. Acta 70 (1986), p. 351-356.

[28] J. L. Heully, I. Lindgren, E. Lindroth, S. Lundqvist, A. M. Mårtensson-Pendrill. Diagonalisation of the Dirac Hamiltonian as a Basis for a Relativistic Many-Body Procedure. J. Phys. B: At. Mol. Phys. 19(1986), p. 2799-2815.

[29] J. L. Heully, I. Lindgren, E. Lindroth, S. Lundqvist, A. M. Mårtensson-Pendrill. Comment on relativistic wave equations and negative-energy states. Phys. Rev. A 33 (1986), p. 4426-4429.

[30] R.N. Hill, C. Krauthauser. A solution to the problem of variational collapse for the oneparticle Dirac equation. Phys. Rev. Lett. 72(14) (1994), p. 2151-2154.

[31] P. Indelicato, J.-P. Desclaux. Projection Operators in the multiconfiguration Dirac-Fock Method. Phys. Scr. T46 (1993), p. 110-114.

[32] W. R. Johnson, S. Blundell, J. Sapirstein. Finite Basis Sets for Dirac Equation Constructed from B splines. Phys. Rev. A 37(2) (1988), p. 307-315. 
[33] S. Komorský, M. Repiský, O. L. Malkina, V. G. Malkin, I. Malkin, M. Kaupp. Resolution of identity Dirac-Kohn-Sham method using the large component only: Calculations of g-tensor and hyperfine tensor. J. Chem. Phys. 124 (2006).

[34] O. Kullie, D. Kolb. High accuracy Dirac-finite-element (FEM) calculations for $\mathrm{H}_{2}^{+}$and $T h_{2}^{179+}$. Eur. Phys. J. D 17 (2) (2001), pp. 167 - 173.

[35] O. Kullie, D. Kolb. Dirac finite element method calculations for $T h_{2}^{179+}$. J. Phys. B: At Mol. Opt. Physics 36 (2003), p. 4361-4366.

[36] O. Kullie, D. Kolb, A. Rutkowski. Two-spinor fully relativistic finite-element (FEM) solution of the two-center Coulomb problem. Chem. Phys. Letters 383 (2004), p. 215-221.

[37] W. Kutzelnigg. Basis Set Expansion of the Dirac Operator without Variational Collapse. Int. J. Quant. Chem. 25 (1984), p. 107-129.

[38] W. Kutzelnigg. Relativistic one-electron Hamiltonians "for electrons only" and the variational treatment of the Dirac equation. Chemical Physics 225 (1997), p. 203-222.

[39] W. Kutzelnigg. Effective Hamiltonians for degenerate and quasidegenerate direct perturbation theory of relativistic effects. J. Chem. Phys. 110(17) (1999), p. 8283-8294.

[40] W. Kutzelnigg, W. Liu. Quasirelativistic theory equivalent to fully relativistic theory. J. Chem. Phys. 123 (2005).

[41] E. van Lenthe, E.J. Baerends, J.G. Snijders. Relativistic regular two-component Hamiltonians. J. Chem. Phys. 99 (1993), p. 4597.

[42] E. van Lenthe, E.J. Baerends, J.G. Snijders. Solving the Dirac equation, using the large component only, in a Dirac-type Slater orbital basis set. Chem. Phys. Lett. 236 (1995), p. 235-241.

[43] M. H. Mittleman. Theory of relativistic effects on atoms: Configuration-space Hamiltonian. Phys.Rev.A 24 (1981), p. 1167.

[44] E. Paturel. Solutions of the Dirac equations without projector. A.H.P. 1 (2000), p. 1123-1157.

[45] W.H.E. Schwarz, H. Wallmeier. Basis set expansions of relativistic molecular wave equations. Mol. Phys. 46(5) (1982), p. 1045-1061.

[46] P. Schwerdtfeger Ed. Relativistic Electronic Structure Theory. Part 1 Fundamental Aspects Elsevier (2002).

[47] R. E. Stanton, S. Havriliak. Kinetic balance: A partial solution to the problem of variational safety in Dirac calculations. J. Chem. Phys. 81 (1984), p. 1910.

[48] B. Swirles. The relativistic self-consistent field. Proc. Roy. Soc. A 152 (1935), p. 625-649.

[49] J.D. Talman. Minimax principle for the Dirac equation. Phys. Rev. Lett. 57(9) (1986), p. 1091-1094.

[50] B. Thaller. The Dirac Equation. Springer-Verlag, 1992.

[51] L. Visscher, K. G. Dyall. Dirac-Fock atomic electronic structure calculations using different nuclear charge distributions. At. Data Nucl. Data Tables, 67 (1997), p. 207.

[52] H. Wallmeier, W. Kutzelnigg. Use of the squared Dirac operator in variational relativistic calculations. Chem. Phys. Lett. 78(2) (1981), p. 341-346.

[53] S. Wilson. On the use of many-body perturbation theory and quantum electrodynamics in molecular electronic structure theory. J. Mol. Struct. (Theochem) 547 (2001), p. 279-291.

[54] J. H. Wood, A. M. Boring. Improved Pauli Hamiltonian for local-potential problems. Phys. Rev. B 18 (1978), p. 2701.

[55] H. Zhang, O. Kullie, D. Kolb. Minimax LCAO approach to the relativistic two-centre Coulomb problem and its finite element (FEM) spectrum. J. Phys. B: At. Mol. Opt. Phys. 37(4) (2004), p. 905-916.

Current address: CEREMADE (UMR CNRS 7534), Université Paris-Dauphine, 75775 Paris Cedex 16, France

E-mail address: esteban@ceremade.dauphine.fr 